\title{
Blocking temperature engineering in exchange-biased CoFeB/IrMn bilayer
}

\author{
Christian Rinaldi ${ }^{1,2}$, Lorenzo Baldrati ${ }^{1}$, Matteo Di Loreto ${ }^{1}$, Marco Asa ${ }^{1}$, Riccardo Bertacco ${ }^{1,2}$, and Matteo Cantoni ${ }^{1}$ \\ ${ }^{1}$ Dipartimento di Fisica, Politecnico di Milano, 20133 Milano, Italy \\ ${ }^{2}$ IFN-CNR, Institute of Photonics and Nanotechnologies, 20133 Milano, Italy
}

\begin{abstract}
In this paper we report on the magnetic and chemical characterization of the exchange-biased CoFeB/IrMn bilayers, grown by magnetron sputtering on a Si-based platform and capped by either a Ru or MgO/Ru overlayer. For Ru capping, the blocking temperature monotonously increases with the IrMn thickness within the investigated range (3.5 - $8 \mathrm{~nm})$. On the contrary, for MgO/Ru capping, the exchange bias is inhibited below $6 \mathrm{~nm}$, whereas above $6 \mathrm{~nm}$ the magnetic behavior is the same of Ru-capped films. The chemical analysis reveals a significant dependence of the Mn content from the capping layer for thin IrMn films (2.5 nm), whereas the difference disappears when IrMn becomes thick $(7 \mathrm{~nm})$. Our work suggests that a non-uniform composition of the IrMn films directly affects the exchange coupling at the IrMn/CoFeB interface.
\end{abstract}

Index Terms - Magnetic and Spintronic Materials, Antiferromagnetic layer, Exchange coupling, Magnetic multilayers.

\section{INTRODUCTION}

The constant need for improving the data storage and manipulation capability of devices is one of the biggest challenges for the research and industry world, which is approaching the limits of Moore's law. Spintronic devices based on ferromagnets (FM), such as modern hard drives or magnetic random access memories (MRAMs), represented a significant improvement in the electronics history [1]. However, the scalability and speed of spintronic devices based on ferromagnetic elements is currently limited by several factors, such as the presence of cross-talk between the layers due to stray fields, the power dissipated during writing and the typical precession frequencies of the ferromagnets in the $\mathrm{GHz}$ range [2]. Antiferromagnetic (AFM) materials are promising candidates for ultrascaled and ultrafast spintronic devices: AFMs do not produce stray magnetic fields, are robust against external unintended magnetic fields and have typical precession frequencies in the $\mathrm{THz}$ range [3]-[5]. Different approaches for storing and reading information in the spin configuration of an AFM layer have been explored. Storing can be achieved by exchange spring with a FM layer [3],[6], field-cooling [7], strain [8], current-induced torques [9] or mechanisms based on the spin Hall Effect [10], whereas reading typically exploits anisotropic magneto-resistance (AMR) effects in thin films [11] or vertical devices comprising a tunnel barrier [3],[7],[12]. Different conducting materials have been investigated for AFM spintronics applications: for example $\mathrm{IrMn}, \mathrm{FeRh}$ [11], $\mathrm{Mn}_{2} \mathrm{Au}$ [13],[14], CuMnAs [15], $\mathrm{Sr}_{2} \mathrm{IrO}_{4}$ [16], and $\mathrm{Cr}$ [17],[18]. Among them, IrMn has long been used as a pinning layer in spintronic devices (GMR, TMR) [19], presents a non-collinear (3Q) spin structure [20] and its AFM phases cover a quite large stoichiometric range [21] in polycrystalline films, at variance with other compounds (e.g., $\mathrm{Mn}_{2} \mathrm{Au}$ and $\mathrm{CuMnAs}$ ) that need a fine tuning of the stoichiometric ratios and very good crystallinity for stabilizing the AFM phase.

Since the observation of tunneling anisotropic magnetoresistance (TAMR) in IrMn/MgO bilayers [7],[22], this antiferromagnet has become an active element for AFM spintronics [9],[23],[24],[25]. For these applications, it is fundamental to understand if the proximity of the $\mathrm{MgO}$ tunneling barrier can alter somehow the structural, chemical or magnetic properties of the thin IrMn film, while trying to act on the IrMn thickness to bring the functionality of the devices towards room temperature (RT). This reason constitutes one driving force of this work.

Another emerging application of IrMn [26] employs IrMn as a platform for creating reconfigurable magnetic landscapes, exploiting local field-cooling performed by an atomic force microscope on a $\mathrm{CoFeB} / \mathrm{IrMn}$ bilayer. This technique opens the route to the development of novel metamaterials with finely tuned magnetic properties, such as reconfigurable magneto-plasmonic and magnonic structures.

For all the applications relying on exchange-biased systems, a control of the blocking temperature is needed. It has to lie above room temperature, but below the maximum temperature that the material can reach in the proximity of the AFM tip. Moreover, the exchange bias at room temperature must be larger than the coercive field.

The dependence of the critical temperature versus the IrMn thickness has been intensively studied in the last decades ([27] and references therein) on several exchange-biased FM/IrMn systems (see, e.g., [26], [28], [29] for Co, CoFe, and NiFe, respectively, playing the role of the FM). The $\mathrm{IrMn} / \mathrm{CoFeB}$ system has already been considered, even if the different heterostructure details and stoichiometry prevent from a strict comparison between the results. In [30], e.g., are reported the exchange bias and coercivity behavior of an $\mathrm{IrMn} / \mathrm{CoFeB}$ system, with the magnetic layer Fe-rich (the stoichiometry is $\mathrm{Co}_{0.2} \mathrm{Fe}_{0.6} \mathrm{~B}_{0.2}$ ) and directly grown on $\mathrm{Si}$. A positive loop shift due to exchange bias is shown, as typically happens in exchange-biased spin valves. A similar behavior has been reported in [31], where $\mathrm{IrMn} / \mathrm{CoFeB}$ is grown on a $\mathrm{CoFe}$ seed layer resulting in a positive exchange-bias even in absence of field-cooling. In some cases, both positive and negative exchange bias coexist [32]. All this variety of possibilities can be ascribed to the polycrystalline nature of IrMn, that makes 
interface (the disordered interface spin structure exhibiting spin-glass-behaviour) and film (the AF grains which undergo thermally activated magnetic reversal) crucial in determining the magnetic properties [33]. The effects of interface composition and stoichiometry on different FM/AFM systems have been addressed too (see, e.g., [34] and [35]).

Here we focus on the $\mathrm{CoFeB} / \mathrm{IrMn}$ bilayer, and we report on its exchange bias properties and critical temperatures versus IrMn thickness and having IrMn in contact with different capping layers $(\mathrm{Ru}$ and $\mathrm{MgO} / \mathrm{Ru}$ ). This work can allow the design of stacks based on this combination of materials suitable for specific applications (e.g. tam-SPL [26] and TAMR [7],[22]) requiring a careful engineering of exchange-bias.

\section{FIG. 1 HERE}

\section{EXPERIMENTAL}

The heterostructures were grown by magnetron sputtering (AJA ATC Orion 8) on $\mathrm{Si}(001) /$ native- $\mathrm{SiO}_{2}$ and $\mathrm{Si}(001) /$ thermal-SiO $2(1 \mu \mathrm{m})$ substrates. In the former, the thin native oxide layer $(\sim 2 \mathrm{~nm})$ is due to the exposure to the air. A magnetic field of $24 \mathrm{kA} / \mathrm{m}$ was applied along $\mathrm{Si}$ [100] during the deposition. The stack was

$\mathrm{Ta}(5) / \mathrm{Ru}(18) / \mathrm{Ta}(3) / \mathrm{CoFeB}(3) / \mathrm{IrMn}\left(t_{\mathrm{IrMn}}\right) / \mathrm{MgO}\left(t_{\mathrm{MgO}}\right) / \mathrm{Ru}\left(t_{\mathrm{Ru}}\right)$ (layer thicknesses in nm), as shown in Fig. 1(a). Metals were grown in dc mode, whereas $\mathrm{MgO}$ was deposited in rf mode. The nominal stoichiometries of $\mathrm{CoFeB}$ and $\mathrm{IrMn}$ targets were $\mathrm{Co}_{0.4} \mathrm{Fe}_{0.4} \mathrm{~B}_{0.2}$ and $\mathrm{Ir}_{0.22} \mathrm{Mn}_{0.78}$ (in the paper, for sake of simplicity we will call them $\mathrm{CoFeB}$ and IrMn, respectively, without specifying the elemental composition). The $\mathrm{Ta}(5) / \mathrm{Ru}(18) / \mathrm{Ta}(3)$ buffer layer was used to avoid $\mathrm{CoFeB}$ intermixing with the substrate, to improve its crystallization [36] and the [111] texture of IrMn, which determines the exchange bias with $\mathrm{CoFeB}$ [37]. The IrMn thickness $\left(t_{\mathrm{IrMn}}\right)$ was varied between 3.5 and $8 \mathrm{~nm}$ to investigate its influence on the IrMn blocking temperature. We also studied the impact of an $\mathrm{MgO}$ interlayer, with thickness compatible with TAMR applications [7],[22] $\left(t_{\mathrm{MgO}}=2.5 \mathrm{~nm}\right)$, between $\mathrm{IrMn}$ and $\mathrm{Ru}$.

The chemical properties have been investigated in ultrahigh vacuum conditions by $\mathrm{X}$-ray Photoemission Spectroscopy (XPS), employing standard unpolarized $\mathrm{Al}-\mathrm{K}_{\alpha}$ and $\mathrm{Mg}-\mathrm{K}_{\alpha} \mathrm{X}$-ray sources and collecting photoelectrons by an Hemispherical Energy Analyzer Phoibos 150 (SPECS $^{\mathrm{TM}}$ ). In samples for XPS the Ru capping layer was thin enough $\left(t_{\mathrm{Ru}}=2 \mathrm{~nm}\right)$ to allow for detection of photoelectrons coming out from IrMn.

The magnetic characterization has been performed by a commercial Vibrating Sample Magnetometer (VSM MicroSense EZ9) on samples with thick Ru capping layer $\left(t_{\mathrm{Ru}}=20 \mathrm{~nm}\right)$ to ensure protection from contamination upon air exposure. The VSM allows for temperature measurements with the sample kept within a nitrogen gas flow. The magnetic behavior of the exchange-biased $\mathrm{CoFeB} / \mathrm{IrMn}$ bilayer was studied to understand how the blocking temperature $\left(T_{\mathrm{B}}\right)$ is affected by the following parameters: (i) the substrate (native vs. thermal oxide); (ii) the IrMn thickness (within the range $3.5-8 \mathrm{~nm})$; (iii) the choice of the overlayer $(\mathrm{MgO} / \mathrm{Ru}$ or $\mathrm{Ru})$. The measurements were carried out by VSM adopting the same protocol for all the samples. First, they were kept for 20 minutes at $T=550 \mathrm{~K}$. Then, a field-cooling (FC) down to $120 \mathrm{~K}$ was performed, with an in-plane magnetic field of $320 \mathrm{kA} / \mathrm{m}$. Subsequently, the hysteresis loops at different increasing temperatures were measured to determine the temperature dependence of the exchange bias.

\section{MAGNETIC CHARACTERIZATION}

Fig. 1(b) reports the hysteresis loops measured after FC on a sample grown on $\mathrm{Si} / \mathrm{SiO}_{2}$, with $t_{\mathrm{rMn}}=3.5 \mathrm{~nm}$ and $\mathrm{Ru}$ capping layer $\left(t_{\mathrm{Ru}}=20 \mathrm{~nm}\right)$. The hysteresis loop progressively shrinks (the coercive field $H_{\mathrm{C}}$ decreases) and shifts (the exchange bias field $H_{\mathrm{EB}}$ decreases) when $T$ increases from $175 \mathrm{~K}$ (red curve, top) to $250 \mathrm{~K}$ (black curve, center) and $325 \mathrm{~K}$ (green curve, bottom).

The exchange bias is negative and the sample presents an inplane uniaxial anisotropy along the FC direction, as expected. In fact, magnetization curves measured in the in-plane perpendicular direction do not show any exchange bias, whereas the out-of-plane direction is a hard magnetization axis due to shape anisotropy (data not shown). The CoFeB film does not acquire perpendicular magnetic anisotropy even for field-cooling of the AFM in the out-of-plane direction.

Two characteristic temperatures for the exchanged-biased system can be extracted from the complete trend versus temperature of $H_{\mathrm{C}}$ and $H_{\mathrm{EB}}$, shown in Fig. 1(c). The first important temperature is the blocking temperature $\left(T_{\mathrm{B}}\right)$, corresponding to the zero of $H_{\mathrm{EB}}$.

Secondly, looking at the coercive field behavior, we define $T^{*}$ as the temperature above which $H_{\mathrm{C}}$ remains constant and is not affected by the presence of the antiferromagnet. According to [38], $T^{*}$ can be assumed to be close to the characteristic Néel temperature $\left(T_{\mathrm{N}}\right)$ of the IrMn film, that is the temperature at which the AFM/paramagnetic transition occurs. For a $\mathrm{CoFeB}(3) / \operatorname{IrMn}(3.5) / \mathrm{Ru}(20)$ heterostructure, $T_{\mathrm{B}}$ and $T^{*}$ result $343 \pm 10 \mathrm{~K}$ and $393 \pm 10 \mathrm{~K}$, respectively.

\section{FIG. 2 HERE}

Fig. 2(a) reports the temperature trend of $H_{\mathrm{EB}}$ of four samples, with the same IrMn thickness $\left(t_{\mathrm{IrMn}}=6 \mathrm{~nm}\right)$ and different capping layer, made by either $R u(20)$ or $\mathrm{MgO}(2.5) / \mathrm{Ru}(20)$, and different substrate, standard $\mathrm{Si}(001)$ or $\mathrm{Si}(001)$ with $\sim 1 \mu \mathrm{m}$ of thermal $\mathrm{SiO}_{2}$ on top $\left(\mathrm{Si} / \mathrm{SiO}_{2}\right.$ from now on). As expected, due to the interposition of the $\mathrm{Ta} / \mathrm{Ru} / \mathrm{Ta}$ buffer layer between the substrate and the rest of the heterostructure, the resulting magnetic properties are relatively insensitive to the choice of standard $\mathrm{Si}(001)$ or $\mathrm{Si} / \mathrm{SiO}_{2}$. On the other hand, it is already evident that the choice of the capping layer $(\mathrm{Ru}$ or $\mathrm{MgO} / \mathrm{Ru})$ strongly influences the behavior and magnitude of the exchange bias versus temperature. 
Fig. 2(b) reports the dependence of $T_{\mathrm{B}}$ and $T^{*}$ on $t_{\mathrm{IrMn}}$ for $\mathrm{Ru}$ and $\mathrm{MgO} / \mathrm{Ru}$ overlayers. The IrMn thickness ranges from $3.5 \mathrm{~nm}$ to $8 \mathrm{~nm}$. When the IrMn layer is covered by Ru, both $T_{\mathrm{B}}$ and $T^{*}$ can be measured down to the lowest IrMn investigated thickness $(3.5 \mathrm{~nm})$ and follow a trend similar to that observed on NiFe/IrMn bilayers by Van Driel et al. [39]. By contrast, the $\mathrm{MgO}$ overlayer inhibits the exchange bias of heterostructures with thin AFM layer $\left(t_{\mathrm{IrMn}}<6 \mathrm{~nm}\right)$ within the investigated temperature range $(100 \mathrm{~K}-500 \mathrm{~K})$, so that $T_{\mathrm{B}}$ and $T^{*}$ cannot be identified. Differently, for thicker IrMn $\left(t_{\mathrm{IrMn}} \geq 6 \mathrm{~nm}\right)$ the exchange bias is observed for both $\mathrm{Ru}$ and $\mathrm{MgO} / \mathrm{Ru}$ overlayers, with the same value of $T_{\mathrm{B}}$ (see also Fig 2(a)) and $T^{*}$, but a different magnitude of $H_{\mathrm{EB}}$. Looking at Fig. 2(a), in this thickness range Ru capped samples present larger $H_{\mathrm{EB}}$ than $\mathrm{MgO} / \mathrm{Ru}$ capped ones. Moreover, $H_{\mathrm{EB}}$ is anyway larger than $H_{\mathrm{C}}$ at $\mathrm{RT}\left(H_{\mathrm{EB}}=15.04 \pm 0.24 \mathrm{kA} / \mathrm{m}\right.$ versus $H_{\mathrm{C}}=13.13 \pm 0.24 \mathrm{kA} / \mathrm{m}$ for $\mathrm{Ru}$ capped samples, and $H_{\mathrm{EB}}=4.30 \pm 0.24 \mathrm{kA} / \mathrm{m}$ versus $H_{\mathrm{C}}=1.59 \pm 0.24 \mathrm{kA} / \mathrm{m}$ for $\mathrm{MgO} / \mathrm{Ru}$ capped samples): this condition is fundamental for the pinning of the ferromagnetic layer as well as for magnetic patterning via local field-cooling.

IrMn layers thicker than $8 \mathrm{~nm}$ were not studied because the expected blocking temperature is higher than the temperature limit at which the heterostructure degrades when prepared by FC $\left(T_{\mathrm{B}}=550 \mathrm{~K}\right.$, extrapolated from Fig. 2(b)). Indeed, we observed an irreversible reduction of both the $\mathrm{CoFeB}$ saturation magnetization and $H_{\mathrm{EB}}$ after annealing above $550 \mathrm{~K}$, probably due to interdiffusion or oxidation.

\section{CHEMICAL CHARACTERIZATION}

In this section, we present the study of IrMn composition as a function of IrMn thickness and overlayers, investigated by means of XPS to gain insight the presented magnetic behavior. The focus is what happens at the buried interface between IrMn and $\mathrm{CoFeB}$ from the chemical point of view, since the magnetic properties discussed above (coercive field, exchange bias field) depend on the exchange coupling between $\mathrm{CoFeB}$ and IrMn at their interface. To this aim, we studied the bunch of samples listed in Table 1, with different heterostructures (IrMn thickness, capping material) and post annealing treatments. The smallest thickness was $2.5 \mathrm{~nm}$, smaller than $3.5 \mathrm{~nm}$ for the magnetic characterization: this is comparable with the electron inelastic mean free paths, aiming at highlighting interface effects.

\section{TABLE 1 HERE}

For each sample, core levels Mn $2 p$ and Ir $4 f$ peaks were acquired by XPS at RT and normal emission. The XPS intensity due to electrons from a core level P (Mn 2p or Ir 4f) is given by

Corresponding author: M. Cantoni (matteo.cantoni@polimi.it) Digital Object Identifier: 10.1109/LMAG.XXXX.XXXXXXX (inserted by IEEE).

$$
I_{\mathrm{P}}=\alpha T_{\mathrm{P}} \sigma_{\mathrm{P}} N_{\mathrm{P}} \lambda_{\mathrm{P}(\mathrm{IrMn})}\left[1-\exp \left(-t_{\text {IrMn }} / \lambda_{\mathrm{P}(\mathrm{IrMn})}\right) \mid \exp \left(-t_{C} / \lambda_{\mathrm{P}(\mathrm{C})}\right)\right.
$$

$\alpha$ is a prefactor accounting for the experimental conditions (photon flux, incidence angle, etc.), $T_{\mathrm{P}}$ is the electron analyzer transmission at a given electron kinetic energy $(K E), \sigma_{\mathrm{P}}$ is the cross section, $N_{\mathrm{P}}$ is the layer atomic density, $\lambda_{\mathrm{P}(\mathrm{IrMn})}$ and $\lambda_{\mathrm{P}(\mathrm{C})}$ are the electron inelastic mean free paths in IrMn and in the capping layer (C) [40], $t_{\mathrm{rMn}}$ and $t_{\mathrm{C}}$ are the IrMn and capping layer thickness, respectively. The Mn (Ir) content is finally calculated as $N_{\mathrm{Mn}(\mathrm{Ir})} /\left(N_{\mathrm{Mn}}+N_{\mathrm{Ir}}\right)$, considering the atomic densities estimated by (1).

Note that the Mn and Ir contents depend, through (1), on the cross-sections and escape depths (in particular through the $\sigma_{\mathrm{P}} \lambda_{\mathrm{P}(\mathrm{IrMn})}$ product) for photoelectrons of the core levels employed for quantification. Since the corresponding $K E$, to which these parameters are related [40], are quite different ( $841 \mathrm{eV}$ for $\mathrm{Mn} 2 \mathrm{p}_{3 / 2}$ versus $\sim 1420 \mathrm{eV}$ for Ir $4 \mathrm{f}$ ), some discrepancies between the real and the estimated products could exist. To give a better estimation of $N_{\mathrm{Mn}}$ and $N_{\text {Ir }}$ through (1), in the following we will instead assume for $\sigma_{\mathrm{Mn}} \lambda_{\mathrm{Mn}(\mathrm{IrMn})}$ and $\sigma_{\mathrm{Ir}} \lambda_{\mathrm{Ir}(\mathrm{IrMn})}$ the values leading to a nominal stoichiometry $\left(\mathrm{Ir}_{22} \mathrm{Mn}_{78}\right)$ for sample U60 (see the Appendix), which represents the uncapped film used for the calibration of the sensitivity factors in XPS.

Equation (1) shows that the compositional information measured by XPS comes from a region of few escape depths $\lambda_{\mathrm{P}(\mathrm{IrMn})}(1.6 \mathrm{~nm}$ for $\mathrm{Mn} 2 \mathrm{p}$ and $2.0 \mathrm{~nm}$ for $\mathrm{Ir} 4 \mathrm{f}$ according to [40]), with a higher sensitivity to the topmost part of the IrMn film and a lower sensitivity to the buried $\mathrm{IrMn} / \mathrm{CoFeB}$ interface. This means that, in the IrMn (2.5) samples, the overall intensity includes some contribution from the $\mathrm{IrMn} / \mathrm{CoFeB}$ interface. For the IrMn (7) samples, instead, the thickness largely exceeds the escape depth and the buried interface is almost inaccessible to XPS investigation. Nevertheless, since the total amount of Mn atoms in a film is determined by its thickness and deposition parameters (pressure, $\mathrm{Ar}$ flow, temperature, rate), a Mn depletion (enrichment) at the topmost surface should correspond an enrichment (depletion) at the $\mathrm{IrMn} / \mathrm{CoFeB}$ interface. Under this assumption, also a surface sensitive technique like XPS can be employed to qualitatively evaluate the Mn depth profile [41].

\section{FIG. 3 HERE}

We first consider sample R25, which presents the higher average Mn content. In Fig. 3(a) we report the XPS spectra of $\mathrm{Mn} 2 \mathrm{p}_{3 / 2}$ and Ir $4 \mathrm{f}$, normalized to the peak areas, measured at normal emission $\left(\theta=0^{\circ}\right.$, black dots) and grazing emission ( $\theta=60^{\circ}$ from the sample normal, red empty dots). The latter, more sensitive to the surface, evidences a larger (smaller) $\mathrm{Mn}$ (Ir) content than the former, more bulk-sensitive, thus indicating the presence of a concentration gradient reducing (increasing) the Mn (Ir) content from top to bottom. This analysis shows the tend ency of Mn diffusion towards the upper interface in samples capped with $\mathrm{Ru}$, which must be associated to a partial $\mathrm{Mn}$ depletion at the $\mathrm{CoFeB} / \mathrm{IrMn}$ 
interface. The same argument applies also to the other samples. In the following a relatively large average $\mathrm{Mn}$ content, as reported in Table 1 , will be assumed as an indication of tendency to $\mathrm{Mn}$ diffusion towards the capping layer and, correspondingly, to partial depletion at the buried $\mathrm{CoFeB} / \mathrm{IrMn}$ interface.

Starting from the thickest (7 nm) IrMn films [R70/R70a (Rucapped) and $\mathrm{M} 70 / \mathrm{M} 70 \mathrm{a}(\mathrm{MgO} / \mathrm{Ru}$-capped)] and considering $\pm 3 \%$ accuracy, we found a slight depletion of $\mathrm{Mn}$ (4-6\%) without any sizeable difference versus capping material $(\mathrm{Ru}$ vs. $\mathrm{MgO} / \mathrm{Ru}$ ) or post-growth annealing. This is coherent with the equivalent magnetic behavior $\left(T_{\mathrm{B}}, T^{*}\right)$ for $t_{\mathrm{IrMn}} \geq 6 \mathrm{~nm}$ shown in Fig. 2 [42]. This points to an intrinsic stability of the $7 \mathrm{~nm}$ thick IrMn layer. Samples with the thinnest IrMn layer (2.5 nm) [R25/R25a (Ru-capped) and M25/M25a ( $\mathrm{MgO} / \mathrm{Ru}$-capped)] show instead a strong influence of the capping layer in determining the $\mathrm{Mn}$ and $\mathrm{Ir}$ content, as shown in Fig. 3(b). According to our discussion above, there is a sizable enrichment (depletion) of interfacial $\mathrm{Mn}$ at the topmost interface with $\mathrm{Ru}(\mathrm{MgO} / \mathrm{Ru})$ capping. The annealing plays a marginal contribution.

\section{FIG. 4 HERE}

Beyond the concentration gradient, the XPS estimation of the Mn concentration could be affected by spurious effects: layers interface and surface roughness, intermixing and/or film oxidation, strain. In order to exclude any relevant role of roughness, we performed Atomic Force Microscopy (AFM) on Ru-capped samples. Fig. 4(a) reports the $10 \mu \mathrm{m} \times 10 \mu \mathrm{m}$ topographic image of the sample R70. The sample is atomically flat, with roughness of $0.3 \mathrm{~nm} \mathrm{(r.m.s.).} \mathrm{The}$ roughness of the sample with thinner IrMn layer (R25) is even lower $(0.25 \mathrm{~nm})$. These findings allow excluding any major role of the roughness, being small and independent on the IrMn thickness. Moreover, $\mathrm{MgO}$ grows layer by layer on flat IrMn surfaces (as previously demonstrated in [7] by some of the authors), so that it cannot be responsible of an increase of roughness. For what concerns intermixing and/or oxidation, Fig. 4(b) reports the Mn $2 \mathrm{p}_{3 / 2}$ peak acquired by XPS on the samples with $2.5 \mathrm{~nm}$ IrMn thickness (for which XPS probes the whole film), and for comparison on the uncapped IrMn sample (U60, see the Appendix). Apart from minor differences, the peak shape is the same, indicating that no relevant oxidation or intermixing takes place. Even if a minor role in determining the $\mathrm{Mn}$ content reported in Fig. 3(b) cannot be excluded for morphology, intermixing and/or oxidation, and strain, we can conclude that the concentration gradient remains the best candidate to explain such a huge variation (about 20\% between M25 and R25). To summarize, we found that there is a one-to-one relationship between chemical (the upper Mn content) and magnetic (presence of exchange bias, critical temperatures) properties, with the exchange bias reinforced when $\mathrm{Mn}$ is pumped towards the uppermost interface. The $7 \mathrm{~nm}$ thick films share the same upper Mn content and all exhibit exchange bias, whereas the $2.5 \mathrm{~nm}$ thick films are strongly affected by the capping layer in both the magnetic and chemical properties, with the exchange bias present (or absent) when the upper Mn content is larger (smaller) with $\mathrm{Ru}(\mathrm{MgO} / \mathrm{Ru})$ capping.

The Mn pumping towards $\mathrm{Ru}$ in thinner films can be ascribed to the formation of a $\mathrm{Mn}-\mathrm{Ru}$ alloy [43]. A possible scenario is the following: when Ru is grown directly on IrMn, the thermal energy released by the sputtered $\mathrm{Ru}$ atoms could activate this reaction, resulting in a tendency of $\mathrm{Mn}$ atoms to be absorbed by, or be close to, the Ru layer. Because Ir atoms are not affected by this process, XPS finds a global increase of the upper $\mathrm{Mn}$ content. When $\mathrm{MgO}$ is placed between $\mathrm{Ru}$ and $\mathrm{MgO}$, this effect is instead inhibited. A more detailed investigation of such mechanisms is anyway beyond the scope of this paper.

A qualitative explanation for the relationship between chemical (the upper Mn content) and magnetic properties could be found in [35], where a direct proportionality between the Ir content and the exchange bias field of a $\mathrm{IrMn} / \mathrm{CoFe}$ bilayer is reported [44]. Considering the samples with $2.5 \mathrm{~nm}$ IrMn thickness, the Ru-capped ones (R25 and R25a) present a negative (positive) Mn (Ir) gradient (see Fig. 3(a)) from top to bottom, whereas for the $\mathrm{MgO} / \mathrm{Ru}$-capped ones (M25 and M25a) the trend is opposite. Following [35], the exchange bias field is predicted to be larger for $\mathrm{Ru}$-capped samples, because the Ir content at the interface with the ferromagnet is larger too, than for $\mathrm{MgO} / \mathrm{Ru}$-capped ones. This behavior reflects our experimental data, with the exchange bias well noticeable for Ru-capped samples and below the experimental accuracy in $\mathrm{MgO} / \mathrm{Ru}$-capped ones.

We can conclude that $\mathrm{Ru}$ plays an active role in preserving the exchange bias, even at very low IrMn thickness, by pumping $\mathrm{Mn}$, and thus leaving the $\mathrm{IrMn} / \mathrm{CoFeb}$ interface Ir-rich. This effect is inhibited when a $\mathrm{MgO}$ interlayer is placed between $\mathrm{Ru}$ and IrMn, and thus the exchange bias disappears. Note that this behavior, that takes place below $6 \mathrm{~nm}$ IrMn thickness in $\mathrm{MgO} / \mathrm{Ru}$-capped samples, could be likely ascribed to the decrease of the blocking temperature below the minimum temperature our system can reach $(100 \mathrm{~K})$. At larger thickness, the $\mathrm{IrMn} / \mathrm{CoFeB}$ interface is no longer influenced by the overlayer and the magnetic behavior becomes independent on it, exhibiting exchange bias both on $\mathrm{Ru}$ and $\mathrm{MgO} / \mathrm{Ru}$ capped samples.

\section{CONCLUSION}

In conclusion, we reported on the magnetic (VSM) and chemical (XPS) characterization of CoFeB/IrMn bilayers, grown by magnetron sputtering on a suitable template $(\mathrm{Ta} / \mathrm{Ru} / \mathrm{Ta})$, with different $\mathrm{IrMn}$ thickness and capping material ( $\mathrm{Ru}$ vs. $\mathrm{MgO} / \mathrm{Ru}$ ). The use of $\mathrm{Si} /$ native- $\mathrm{SiO}_{2}$ or $\mathrm{Si} /$ thermal- $\mathrm{SiO}_{2}(1 \mu \mathrm{m})$ substrates does not influence the magnetic properties of the $\mathrm{IrMn} / \mathrm{CoFeB}$ bilayer, probably thanks to the buffer template $\mathrm{Ta} / \mathrm{Ru} / \mathrm{Ta}$. The critical

Corresponding author: M. Cantoni (matteo.cantoni@polimi.it) Digital Object Identifier: 10.1109/LMAG.XXXX.XXXXXXX (inserted by IEEE). 
temperatures (the blocking temperature and $T^{*}$ ) increase vs. the IrMn thickness but, whereas for Ru capping this is true in the whole thickness range we investigated $(3.5-8 \mathrm{~nm})$, for $\mathrm{MgO} / \mathrm{Ru}$ capping exchange bias is inhibited below $6 \mathrm{~nm}$, whereas from $6 \mathrm{~nm}$ the behavior is the same of Ru-capped films. The Mn content depends even on the capping layer (the $\mathrm{Mn}$ content close to the interface with $\mathrm{CoFeB}$ is smaller (larger) than the bulk-like situation for $\mathrm{Ru}(\mathrm{MgO} / \mathrm{Ru})$ capping) in thin films $(2.5 \mathrm{~nm})$, whereas is independent in thicker films $(7 \mathrm{~nm})$. Our work suggests that a non-uniform composition of the IrMn films directly affects the exchange coupling at the $\mathrm{IrMn} / \mathrm{CoFeB}$ interface.

Table 1. List of the samples investigated by XPS. The capital letter refers to the capping material ( $U=$ uncapped, $\mathrm{R}=\mathrm{Ru}, \mathrm{M}=\mathrm{MgO}$ ), the number is the IrMn thickness in Angstrom, and the final letter (a), if present, indicates that the sample has been annealed. The $\mathrm{Mn}$ content is calculated as $N_{\mathrm{Mn}} /\left(N_{\mathrm{Mn}}+N_{\mathrm{Ir}}\right)$, where $N_{\mathrm{Mn}}$ and $N_{\text {Ir }}$ are the Mn and Ir atomic densities estimated by (1).

\begin{tabular}{ccccc}
$\begin{array}{c}\text { Sample } \\
\text { label }\end{array}$ & $\begin{array}{c}\text { IrMn } \\
\text { thickness }\end{array}$ & $\begin{array}{c}\text { IrMn } \\
\text { overlayer }\end{array}$ & $\begin{array}{c}\text { Post- } \\
\text { growth } \\
\text { annealing }\end{array}$ & Mn content \\
\hline $\mathrm{U} 60$ & $6 \mathrm{~nm}$ & $\mathrm{none}$ & $\mathrm{no}$ & $78 \%$ \\
$\mathrm{R} 25$ & $2.5 \mathrm{~nm}$ & $\mathrm{Ru}$ & no & $92 \%$ \\
$\mathrm{R} 25 \mathrm{a}$ & $2.5 \mathrm{~nm}$ & $\mathrm{Ru}$ & $523 \mathrm{~K}$ & $95 \%$ \\
$\mathrm{R} 70$ & $7 \mathrm{~nm}$ & $\mathrm{Ru}$ & no & $72 \%$ \\
$\mathrm{R} 70 \mathrm{a}$ & $7 \mathrm{~nm}$ & $\mathrm{Ru}$ & $523 \mathrm{~K}$ & $73 \%$ \\
$\mathrm{M} 25$ & $2.5 \mathrm{~nm}$ & $\mathrm{MgO} / \mathrm{Ru}$ & no & $65 \%$ \\
$\mathrm{M} 25 \mathrm{a}$ & $2.5 \mathrm{~nm}$ & $\mathrm{MgO} / \mathrm{Ru}$ & $523 \mathrm{~K}$ & $68 \%$ \\
$\mathrm{M} 70$ & $7 \mathrm{~nm}$ & $\mathrm{MgO} / \mathrm{Ru}$ & no & $74 \%$ \\
$\mathrm{M} 70 \mathrm{a}$ & $7 \mathrm{~nm}$ & $\mathrm{MgO} / \mathrm{Ru}$ & $523 \mathrm{~K}$ & $74 \%$
\end{tabular}
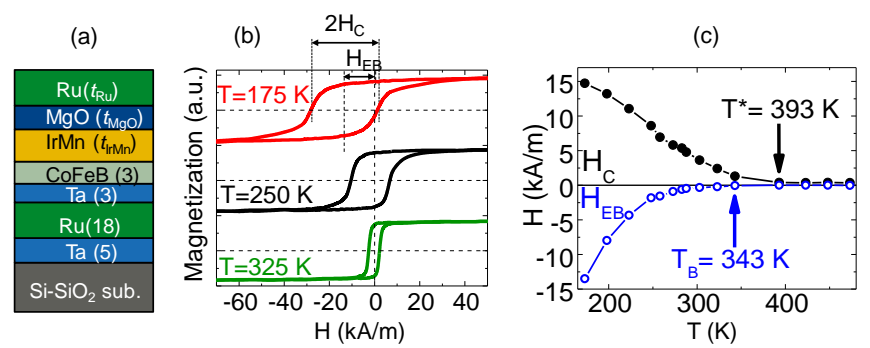

Fig. 1. (a) sketch of the heterostructure; (b) hysteresis loops of a $\mathrm{Si} / \mathrm{SiO}_{2} / \mathrm{Ta}(5) / \mathrm{Ru}(18) / \mathrm{Ta}(3) / \mathrm{CoFeB}(3) / \mathrm{IrMn}(3.5) / \mathrm{Ru}(20)$ heterostructure after field-cooling; (c) temperature dependence of the coercive field $\left(H_{\mathrm{C}}\right)$ and the exchange bias field $\left(H_{\mathrm{EB}}\right) . T^{*}$ and $T_{\mathrm{B}}$ are defined as the temperatures at which $H_{\mathrm{C}}$ (black curve, top) and $H_{\mathrm{EB}}$ (blue curve, bottom), respectively, approach to asymptotical values (zero in the case of $H_{\mathrm{EB}}$ ).
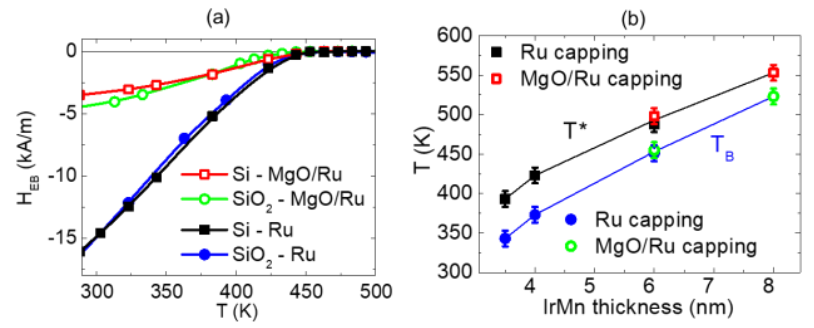

Fig. 2. (a) Exchange bias field $\left(H_{\mathrm{EB}}\right)$ as a function of the temperature for samples with $t_{\mathrm{rMn}}=6 \mathrm{~nm}$, grown on either $\mathrm{Si}$ or $\mathrm{Si} / \mathrm{SiO}_{2}$ substrates, with Ru or $\mathrm{MgO} / \mathrm{Ru}$ capping; (b) blocking temperature $\left(T_{\mathrm{B}}\right)$ and $T^{*}$ as a function of the IrMn thickness for different overlayers $(\mathrm{MgO} / \mathrm{Ru}$ or $\mathrm{Ru})$.
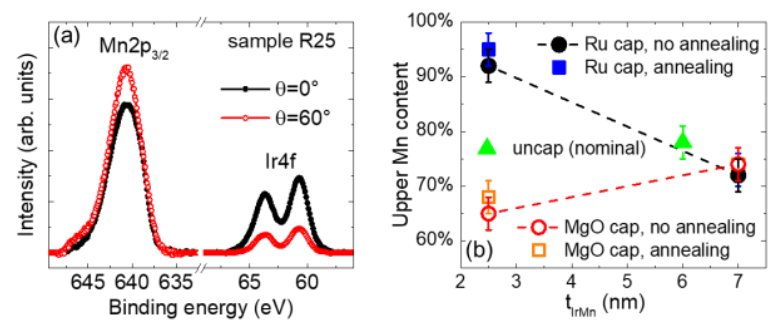

Fig. 3. (a) XPS spectra on Mn $2 p_{3 / 2}$ and Ir $4 \mathrm{f}$ core levels on sample R25 at normal $\left(\theta=0^{\circ}\right.$, black dots) and grazing $\left(\theta=60^{\circ}\right.$, red empty dots) emission, evidencing a Mn surface segregation; (b) Mn content measured by XPS vs. IrMn thickness, evaluated by XPS measuring Mn $2 \mathrm{p}_{3 / 2}$ and Ir $4 \mathrm{f}$ peaks, for the samples reported in Table 1 . The dashed lines are only guides for the eye. (a)

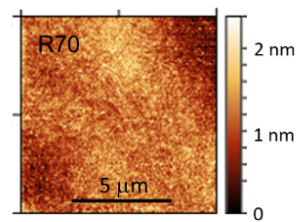

(b)

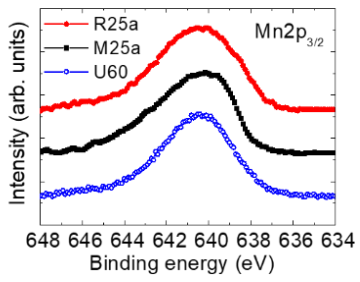

Fig. 4. (a) AFM of R70 sample, $10 \mu \mathrm{m} \times 10 \mu \mathrm{m}$ area. The roughness r.m.s. is $0.3 \mathrm{~nm}$. (b) XPS spectra on Mn 2 $\mathrm{p}_{3 / 2}$ core level on samples R25, R70, and $\mathrm{U} 60$ at normal emission.

\section{APPENDIX}

\section{A. Chemical characterization of IrMn uncapped films}

Two uncapped IrMn films, with $t_{\mathrm{IrMn}}=6 \mathrm{~nm}$ (sample U60) and $t_{\mathrm{IrMn}}=20 \mathrm{~nm}$ (sample $\mathrm{U} 200$ ) respectively, were used as reference for XPS characterization of the IrMn films discussed in the paper (see Table 1). The base template was $\mathrm{Si}(001) /$ thermal- $\mathrm{SiO}_{2}(1 \mu \mathrm{m}) / \mathrm{Ta}(5 \mathrm{~nm}) / \mathrm{Ru}(18 \mathrm{~nm}) / \mathrm{Ta}(3 \mathrm{~nm})$.

Mn 3p, Mn 2p, Ir 4f and O 1s peaks were acquired by XPS at room temperature and normal incidence employing $\mathrm{Al}-\mathrm{K}_{\alpha}$ and $\mathrm{Mg}-\mathrm{K}_{\alpha}$ sources. The corresponding electron escape depths are well below $t_{\mathrm{IrMn}}$ for both the samples [40]: any spurious contribution due to the interface with the bottom layer $(\mathrm{CoFeB})$ can thus be excluded in the IrMn analysis.

Looking at the stoichiometry, the relative concentrations of Ir and $\mathrm{Mn}$ were initially measured by acquiring Mn $3 p$ and Ir $4 \mathrm{f}$ peaks with $\mathrm{Al}-\mathrm{K}_{\alpha}$ source. We choose these peaks because of the small difference in the photoelectron kinetic energies $(K E)$, and thus in the corresponding electron escape depths, meaning the IrMn region probed by XPS is almost the same. The photoelectron intensity from the peak P (Mn 3p or Ir 4f) is given by:

$$
I_{\mathrm{P}}=\alpha T_{P} \sigma_{\mathrm{P}} N_{\mathrm{P}} \lambda_{\mathrm{P}}\left[1-\exp \left(-t_{I r M n} / \lambda_{\mathrm{P}}\right)\right]
$$

$\alpha$ is a prefactor accounting for experimental conditions (photon flux, incidence angle, etc.), $T_{\mathrm{P}}$ is the electron analyzer transmission, $\sigma_{\mathrm{P}}$ is the atomic cross section, $N_{\mathrm{P}}$ is the atomic 
density, $\lambda_{\mathrm{P}}$ is the escape depth, and $t_{\mathrm{IrMn}}$ is the IrMn film thickness. The Mn [Ir] content has been calculated as $N_{\mathrm{Mn}} /\left(N_{\mathrm{Mn}}+N_{\mathrm{Ir}}\right)\left[N_{\mathrm{Ir}} /\left(N_{\mathrm{Mn}}+N_{\mathrm{Ir}}\right)\right]$, and resulted $79 \%$ [21\%] for sample U60 and 78\% [22\%] for sample U200. Within the experimental accuracy $( \pm 3 \%)$, the two samples appear identical and coherent with the target composition $\left(\operatorname{Ir}_{22} \mathrm{Mn}_{78}\right)$.

\section{FIG. 1A HERE}

In Fig. (1A) we report the $\mathrm{Mn} 2 \mathrm{p}$ and $\mathrm{O} 1 \mathrm{~s}$ peaks from sample $\mathrm{U} 60$ at room temperature, measured by $\mathrm{Al}-\mathrm{K}_{\alpha}$ and $\mathrm{Mg}-\mathrm{K}_{\alpha}$ sources, respectively, after subtracting an integral background due to secondary electrons. Mn $2 \mathrm{p}_{3 / 2}$ in panel (a) presents a shoulder, with binding energy $5.7 \mathrm{eV}$ larger than the main peak one. Following [45], this can be ascribed to a $\mathrm{Mn}$ oxidation state with +2 valence, possibly due to oxygen coming from air exposure during the transfer from growth to measurement chambers. The presence of the oxygen is also confirmed by the $\mathrm{O} 1 \mathrm{~s}$ spectrum, reported in panel (b). In this case, two contributions can be clearly distinguished: a larger one at higher binding energy (dashed red line) and a smaller one at lower binding energy (dashed-dotted blue line). If sample is tilted by $60^{\circ}$ with respect to the normal (in order to increase the surface sensitivity), the former increases and the latter decreases. This allows us to ascribe the first contribution, centered at $532 \mathrm{eV}$, to atomic oxygen lying on the IrMn surface and thus evidenced at grazing incidence, and the second, centered at $529.8 \mathrm{eV}$, to oxygen bonded with $\mathrm{Mn}$ inside the film [46]. The ratio between the areas of the film and surface components $\left(R_{\mathrm{F} / \mathrm{S}}\right)$ decreases from 0.8 at normal incidence to 0.4 at grazing incidence $\left(60^{\circ}\right.$ from the normal, data not shown). We note that, despite the peak and shoulder positions suggest a $+2 \mathrm{Mn}$ oxidation state [45], other situations cannot be excluded. Actually, tabulated shapes and positions do not strictly apply to our case, because they come from Mn oxides and hydroxides, whereas the Ir contribution could play a role in determining position and shape of the core lines.

At variance with $\mathrm{Mn}$, Ir $4 \mathrm{f}$ does not show any oxidation feature. This can be explained on the basis of the enthalpy of formation of the respective compounds [47]: because all Mn oxides are energetically favourable with respect to Ir oxides [48], oxygen embedded in the IrMn matrix will preferably bind to Mn, leaving Ir not oxidized.

Finally, the effect of an annealing at $523 \mathrm{~K}$ for 30 minutes has been evaluated. This is a typical procedure employed in IrMn-based devices for setting exchange bias properties by a field-cooling procedure. The stoichiometry of both samples is unaffected by this procedure (Mn content increases of $1 \%$, smaller than the $\pm 3 \%$ accuracy). The ratio $R_{\mathrm{F} / \mathrm{S}}$ instead more than doubles after annealing, probably because of thermal desorption of surface oxygen. Anyway, peak positions of both film and surface components do not change, indicating that there is no chemical modification of the structure.

Finally, it is worthwhile to note that, if we employ Mn $2 p$ instead of Mn 3p for Mn quantification (as in the paper), an apparent Mn-deficiency appears, with 69\% Mn concentration instead of $78 \%\left(\operatorname{Ir}_{31} \mathrm{Mn}_{69}\right.$ instead of $\left.\operatorname{Ir}_{22} \mathrm{Mn}_{78}\right)$. This can be due to an incorrect estimation of the Mn $2 p$ electron escape depth (we assumed $1.6 \mathrm{~nm}$ following [40], coherently with the other peaks): as a matter of fact, over(under)estimation of this parameter would predict a smaller (larger) Mn content. The better reliability of $\mathrm{Mn} 3 \mathrm{p}$, as proved by the coherency with the nominal target stoichiometry, can be ascribed to the proximity of $\mathrm{Mn} \mathrm{3p}$ and Ir 4f peaks (the binding energies are $48 \mathrm{eV}$ and 61-64 eV, respectively, whereas $\mathrm{Mn} \mathrm{2} \mathrm{p}_{3 / 2}$ is at $641 \mathrm{eV}$ ), resulting in electron escape depths more similar, and thus almost identical probed depths. Unfortunately, Mn 3p cannot be used in $\mathrm{Ru}$ and $\mathrm{MgO} / \mathrm{Ru}$ capped samples, because of the superposition with the $\mathrm{Ru} 4 \mathrm{p}$ peak: then, in the analysis reported in the paper we have been forced to use Mn $2 p$ and employ a normalization procedure in order to evaluate the real stoichiometry.
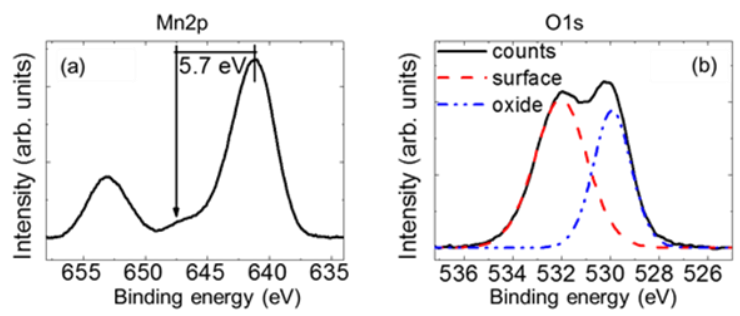

Fig. 1A. (a) Mn 2p and (b) O 1s features from sample B at room temperature, measured by $\mathrm{Al}-\mathrm{K}_{\alpha}$ and $\mathrm{Mg}-\mathrm{K}_{\alpha}$ sources respectively, after subtracting an integral background due to secondary electrons.

\section{ACKNOWLEDGMENT}

We thank D. Petti and E. Albisetti for fruitful discussion, and S. Bertoli for supporting sample growth and characterization. The authors gratefully acknowledge financial support from Fondazione Cariplo via the project Magister (grant n. 2013-0726). This work was partially performed at Polifab, the micro and nanofabrication facility of Politecnico di Milano.

\section{REFERENCES}

[1] G. Varvaro and F. Casoli, Ultra-high-density magnetic recording: storage materials and media designs, Pan Stanford, 2016, pp. 457-509.

[2] M. Krounbi et al., "Status and Challenges in Spin-Transfer Torque MRAM Technology", Proc. 228th ECS Meet. vol. 69, pp. 119-126, 2015.

[3] E. V. Gomonay and V. M. Loktev, "Spintronics of antiferromagnetic systems", Low Temp. Phys. vol. 40, p. 17, 2014.

[4] T. Jungwirth, X. Marti, P. Wadley, and J. Wunderlich, "Antiferromagnetic spintronics", Nature Nanotech. Vol. 11, pp. 231241, 2016.

[5] T. Seifert et al., "Efficient metallic spintronic emitters of ultrabroadband terahertz radiation", Nature Photonics vol. 10, pp. 483-488, 2016.

[6] Y. Y. Wang, C. Song, G. Y. Wang, F. Zeng, and F. Pan, "Evidence for asymmetric rotation of spins in antiferromagnetic exchange-spring", New Journal of Physics vol. 16, p. 123032, 2014.

[7] D. Petti et al., "Storing magnetic information in $\mathrm{IrMn} / \mathrm{MgO} / \mathrm{Ta}$ tunnel junctions via field-cooling”, Appl. Phys. Lett. vol. 102, p. 192404, 2013. 
[8] A. A. Sapozhnik et al., "Manipulation of antiferromagnetic domain distribution in $\mathrm{Mn}_{2} \mathrm{Au}$ by ultrahigh magnetic fields and by strain", Rapid Research Letters vol. 11, p. 1600438, 2017.

[9] H. Reichlova et al., "Current-induced torques in structures with ultrathin IrMn antiferromagnets", Phys. Rev. B vol. 92, p. 165424, 2015.

[10] A. van der Brink et al., "Field-free magnetization reversal by spin-Hall effect and exchange bias", Nature Comm. vol. 7, p. 10854, 2016.

[11] X. Marti et al., "Room-temperature antiferromagnetic memory resistor", Nature Mat. vol. 13, p. 367, 2014.

[12] A. B. Shick, S. LKhmelevskyi, O. N. Mryasov, J. Wunderlich, and T. Jungwirth, "Spin-orbit coupling induced anisotropy effects in bimetallic antiferromagnets: A route towards antiferromagnetic spintronics", Phys. Rev. B vol. 81, p. 212409, 2010.

[13] V. M. T. S. Barthem, C. V. Colin, H. Mayaffre, M.-H. Julien, and D. Givord, "Revealing the properties of $\mathrm{Mn}_{2} \mathrm{Au}$ for antiferromagnetic spintronics", Nature Comm. vol. 4, p. 2892, 2013.

[14] E. Plekhanov, A. Stroppa, and S. Picozzi, "Magneto-electric coupling in antiferromagnet/ferroelectric $\mathrm{Mn}_{2} \mathrm{Au} / \mathrm{BaTiO}_{3}$ interface", J. Appl. Phys. vol. 120, p. 074104, 2016

[15] P. Wadley et al., "Electrical switching of an antiferromagnet", Science vol. 351, pp. 587-590, 2016.

[16] I. Fina et al., "Anisotropic magnetoresistance in an antiferromagnetic semiconductor", Nature Comm. vol. 5, p. 4671, 2014.

[17] W. B. Muir and J. O. Strom-Olsen, "Electrical Resistance of SingleCrystal Single-Domain Chromium from 77 to 325 K", Phys. Rev. B vol. 4, p. 988, 1971

[18] M. Cantoni et al., "Towards Cr-based antiferromagnetic spintronics: growth and magnetic anisotropy of Chromium thin films", conference acta of AIM 2016, Bormio, Italy, 14-16 March 2016

[19] S. S. Parkin et al., "Giant tunnelling magnetoresistance at room temperature with $\mathrm{MgO}$ (100) tunnel barriers", Nature Mat. vol. 3, p. 862, 2004.

[20] A. Kohn et al., "The antiferromagnetic structures of IrMn3 and their influence on exchange-bias", Scientific Rep. vol. 3, p. 2412, 2013.

[21] H. Okamoto, "The Ir-Mn (Iridium-Manganese) System", Journal of Phase Equilibria vol. 17, pp. 60-62, 1996.

[22] B. G. Park et al., "A spin-valve-like magnetoresistance of an antiferromagnet-based tunnel junction", Nature Mat. vol. 10, pp. 347-351, 2011

[23] L. Frangou et al., "Enhanced Spin Pumping Efficiency in Antiferromagnetic IrMn Thin Films around the Magnetic Phase Transition", Phys. Rev. Lett. vol. 116, p. 077203, 2016.

[24] S. Rizwan, G. Q. Yu, S. Zhang, Y. G. Zhao, and X. F. Han, "Electricfield control of $\mathrm{CoFeB} / \mathrm{IrMn}$ exchange bias system", J. Appl. Phys. vol. 112, p. 064120, 2012.

[25] W. J. Kong et al., "Field-free spin Hall effect driven magnetization switching in $\mathrm{Pd} / \mathrm{Co} / \mathrm{IrMn}$ exchange coupling system", Appl. Phys. Lett. vol. 109 p. 132402, 2016.

[26] E. Albisetti et al., "Nanopatterning reconfigurable magnetic landscapes via thermally assisted scanning probe lithography", Nature Nanotech. vol. 11, pp. 545-551, 2016.

[27] J. Nogués and I. K. Schuller, "Exchange bias", J. Magn. Magn. Mat. vol. 192, pp. 203-232, 1999.

[28] S. S. Lee, J. G. Choi, S. W. Kim, J. R. Rhee, and D. G. Hwang, "Antiferromagnet IrMn thickness dependence in exchange-biased perpendicular magnetic anisotropy based on $\mathrm{CoFe} / \mathrm{Pt} / \mathrm{CoFe} /\left[\mathrm{IrMn}\left(\mathrm{t}_{\mathrm{IrMn}}\right)\right]$ multilayers", J. Magn. and Magn. Mat. vol. 304, pp. e91-e93, 2006.

[29] A. J. Devasahayam and M. H. Kryder, "The dependence of the antiferromagnet/ferromagnet blocking temperature on antiferromagnet thickness and deposition conditions", J. Appl. Phys. vol. 85, p. 5519, 1999.

[30] M. Raju, S. Chaudhary, and D. K. Pandya, “ Magnetic annealing of the ion-beam sputtered $\mathrm{IrMn} / \mathrm{CoFeB}$ bilayers - Positive exchange bias with reduced coercivity", Eur. Phys. J. B vol. 86, p. 491, 2013.

[31] H. Fulara, S. Chaudhary, S. C. Kashyap, and D. K. Pandya, "Positive Exchange Bias in Ion-Beam Sputtered $\mathrm{IrMn} / \mathrm{CoFeB}$ System Grown on CoFe Seed Layer", Nanoscience and Nanotechnology Letters vol. 4, pp. 651-655 (2012)

[32] S. Anandakumar, V. Sudha Rani, S. Oh, C. Kim, "Positive and negative exchange bias in IrMn/NiFe bilayers", Thin Solid Films vol. 519, pp. 1020-1024, 2010.

[33] V. Baltz, B. Rodmacq, A. Zarefy, L. Lechevallier, and B. Dieny, "Bimodal distribution of blocking temperature in exchange-biased ferromagnetic/antiferromagnetic bilayers", Phys. Rev. B vol. 81, p. 052404, 2010.
[34] Y. Tsuchiya, K. Kosuge, and S. Yamaguchi, "Exchange anisotropy of $\mathrm{CrNx} / \mathrm{FeNy} / \mathrm{CrNx}$ trilayer thin films prepared by Reactive Sputtering", Material Transactions, JIM, vol. 38, pp. 91-98, 1997.

[35] N. P. Aley and K. O'Grady, "Compositional dependence of antiferromagnetic anisotropy in $\mathrm{IrMn} / \mathrm{CoFe}$ exchange bias systems", $J$. Appl. Phys. vol. 109, p. 07D719, 2011.

[36] J. Cao, J. Kanak, T. Stobiecki, P. Wisniowski, and P. P. Freitas, "Effect of Buffer Layer Texture on the Crystallization of $\mathrm{CoFeB}$ and on the Tunnel Magnetoresistance in $\mathrm{MgO}$ Based Magnetic Tunnel Junction", IEEE Transactions on Magnetics vol. 45, pp. 3464-3466, 2009.

[37] H. R. Liu et al., "The optimization of Ta buffer layer in magnetron sputtering IrMn top spinvalve", Thin solid Films vol. 441, pp. 111-114, 2003

[38] M. Ali et al., "Antiferromagnetic layer thickness dependence of the IrMn/Co exchange-bias system", Phys.Rev. B vol. 68, p. 214420, 2003.

[39] J. van Driel, F. R. de Boer, K.-M. H. Lenssen, and R. Coehoorn, "Exchange biasing by $\mathrm{Ir}_{19} \mathrm{Mn}_{81}$ : Dependence on temperature, microstructure and antiferromagnetic layer thickness", J. Appl. Phys. vol. 88, p. $975,2000$.

[40] M. P. Seah and W. A. Dench, "Quantitative electron spectroscopy of surfaces: A standard data base for electron inelastic mean free paths in solids", Surf. and Interf. Analysis vol. 1, pp. 2-11, 1979.

[41] The only assumption we make is that the Mn content changes monotonically along the film depth.

[42] The critical temperatures for these films result $T^{*}=488 \pm 15 \mathrm{~K}$ and $T_{\mathrm{B}}=523 \pm 15 \mathrm{~K}$, from Fig. 2 .

[43] M. Jahnatek et al., "Ordered phases in ruthenium binary alloys from high-throughput first-principles calculations", Phys. Rev. B vol. 84, p. 214110, 2011.

[44] We assume that the exchange bias field increases with the Ir content, as in the left part of Fig. 3 in [35]. This is clearly true for $\mathrm{Ru} / \mathrm{MgO}$-capped samples.

[45] M. C. Biesinger et al., "Resolving surface chemical states in XPS analysis of first row transition metals, oxides and hydroxides: $\mathrm{Cr}, \mathrm{Mn}$, Fe, Co and Ni", Appl. Surf. Sci. vol. 257, p. 2717, 2011.

[46] We tried to remove the surface contamination by sputtering, but, at the IrMn level, the different cross sections of Mn and Ir completely altered the IrMn stoichiometry (the Mn etching rate is about 1.6 times the Ir one).

[47] H.J.T. Ellingham, "Reducibility of oxides and sulphides in metallurgical processes", J. Soc. Chem. Ind.vol. 63, pp. 125-133, 1944.

[48] The enthalpy of formation for $\mathrm{IrO}_{2}$ is $\Delta H=-274.1 \mathrm{~kJ} / \mathrm{mol}$, to be compared with $\Delta H=-385.2 \mathrm{~kJ} / \mathrm{mol}$ of $\mathrm{MnO},-520.0 \mathrm{~kJ} / \mathrm{mol}$ of $\mathrm{MnO}_{2},-959.0 \mathrm{~kJ} / \mathrm{mol}$ of $\mathrm{Mn}_{2} \mathrm{O}_{3}$ and $-1387.8 \mathrm{~kJ} / \mathrm{mol}$ of $\mathrm{Mn}_{3} \mathrm{O}_{4}$, from D. R. Lide, $C R C$ Handbook of Chemistry and Physics, CRC Press, 2013.

Christian Rinaldi graduated in Physics engineering in 2009 and received the Ph.D. degree in Physics from Politecnico di Milano in 2013.

$\mathrm{He}$ is currently an assistant professor with the Physics Engineering Department, Politecnico di Milano, Italy. He has been visiting researcher at the Institute of Physics of Czech Academy of Science. His current activity focuses on the electric control of spin phenomena in spintronic devices based on semiconductors, (anti-)ferromagnets, functional oxides and their combinations. He is co-author of about 30 scientific papers.

Matteo Cantoni graduated in Nuclear Engineering in 1999 and received the Ph.D. degree in Physics from Politecnico di Milano in 2005.

$\mathrm{He}$ is currently an Associate Professor with the Physics Engineering Department, Politecnico di Milano, Italy. His current research interests include antiferromagnet spintronics, semiconductor spintronics and multiferroic materials for IT and neuromorphic applications. He is co-author of about 70 scientific papers and 4 patent applications. 